\title{
Germ cell nests in adult ovaries and an unusually large ovarian reserve in the naked mole-rat
}

\author{
Ned J Place ${ }^{1}$, Alexandra M Prado', Mariela Faykoo-Martinez², Miguel Angel Brieño-Enriquez³, \\ David F Albertini ${ }^{4}$ and Melissa $M$ Holmes $^{2,5}$ \\ ${ }^{1}$ Department of Population Medicine \& Diagnostic Sciences, Cornell University, Ithaca, New York, USA, \\ ${ }^{2}$ Department of Cell \& Systems Biology, University of Toronto, Toronto, Ontario, Canada, ${ }^{3}$ Department of Obstetrics, \\ Gynecology \& Reproductive Medicine, Magee-Womens Research Institute, University of Pittsburgh, Pittsburgh, \\ Pennsylvania, USA, ${ }^{4}$ Department of Reproductive Biology, Bedford Research Foundation, Bedford, Massachusetts, \\ USA and ${ }^{5}$ Department of Psychology, University of Toronto Mississauga, Mississauga, Ontario, Canada
}

Correspondence should be addressed to N J Place; Email: njp27@cornell.edu

\begin{abstract}
The naked mole-rat (NMR, Heterocephalus glaber) is renowned for its eusociality and exceptionally long lifespan (> 30 y) relative to its small body size (35-40 g). A NMR phenomenon that has received far less attention is that females show no decline in fertility or fecundity into their third decade of life. The age of onset of reproductive decline in many mammalian species is closely associated with the number of germ cells remaining at the age of sexual maturity. We quantified ovarian reserve size in NMRs at the youngest age ( 6 months) when subordinate females can begin to ovulate after removal from the queen's suppression. We then compared the NMR ovarian reserve size to values for 19 other mammalian species that were previously reported. The NMR ovarian reserve at 6 months of age is exceptionally large at 108,588 $\pm \mathbf{6 9 , 8 9 0}$ primordial follicles, which is more than 10-fold larger than in mammals of a comparable size. We also observed germ cell nests in ovaries from 6-month-old NMRs, which is highly unusual since breakdown of germ cell nests and the formation of primordial follicles is generally complete by early postnatal life in other mammals. Additionally, we found germ cell nests in young adult NMRs between 1.25 and 3.75 years of age, in both reproductively activated and suppressed females. The unusually large NMR ovarian reserve provides one mechanism to account for this species' protracted fertility. Whether germ cell nests in adult ovaries contribute to the NMR's long reproductive lifespan remains to be determined.
\end{abstract}

Reproduction (2021) 161 89-98

\section{Introduction}

Reproductive lifespan in female mammals, both within and among species, is largely dependent on the size of the ovarian reserve of germ cells at the age when individuals become sexually mature (Gosden et al. 1983, Gosden \& Telfer 1987). Thereafter, the rates of germ cell attrition and reduced oocyte quality, as well as aging-associated decline in hypothalamic-pituitary function, are additional factors that influence the timing of reproductive senescence (reviewed in Gore et al. 2015). Events that occur long before pubertal onset can have major impacts on the size of the ovarian reserve at sexual maturity. For example, the number of primordial germ cells (PGCs) that successfully migrate from the embryonic epiblast to the genital ridges during early embryonic development has an effect on the ovarian reserve, as does PGC mitotic activity during migration (Funkuda 1976, Findlay et al. 2015, Gomes Fernandes et al. 2018). Continued mitotic activity of PGCs and oogonia within the developing ovary similarly contribute to the maximum number of female germ cells, which is typically achieved during fetal life - embryonic days 13.5-15.5 (E13.5-E15.5) in mice (Myers et al. 2014). In humans, during the ninth week post-fertilization, proliferating PGCs begin to differentiate into oogonia, which have higher mitotic activity than PGCs. This activity continues to 20 weeks of gestation (Motta et al. 1997, Pereda et al. 2006, Mamsen et al. 2011). As female germ cells transition from mitotic activity to the initiation of meiosis and formation of germ cell nests, the first major wave of germ cell apoptosis gets undeway (Coucouvanis et al. 1993). This occurs at E13.5-E15.5 in mice and at 10-12 weeks post-fertilization in humans.

A second and more pronounced wave of germ cell loss occurs when nests break down and individual primordial follicles (PFs) form (Pepling 2006, Rodrigues et al. 2009). The formation of PFs, which are composed of an oocyte surrounded by flat pre-granulosa cells, is essentially complete by postnatal 5 (P5) in mice. In humans, PF formation begins at 17-20 weeks gestation and continues until term (Motta \& Makabe 1986a, b, Motta 
et al. 1997), followed by a $39 \%$ decline in the number of PFs between birth and puberty (Wallace \& Kelsey 2010). In mice, during the interval between P5 and sexual maturity, approximately half of the non-growing PFs are lost due to atresia and when they transition to growing, primary follicles (Tingen et al. 2009). This despite the fact that these growing follicles have no chance of being fertilized and contributing to individual fitness. In the absence of a mature gonadotropin milieu, follicle growth is arrested well before the peri-ovulatory stage, which leads to follicle atresia and continuous declines in the size of the ovarian reserve of PFs (reviewed by Forabosco \& Sforza 2007, Monget et al. 2012, Grive \& Freiman 2015, Ge et al. 2019).

The size of the ovarian reserve at sexual maturity shows substantial variability both within females of the same species and among different species of mammals. The intra- and inter-species differences in ovarian reserve size are thought to be major contributors to differences in female reproductive longevity (Finch \& Kirkwood 2000). Another critical factor that is likely associated with reproductive longevity is adult lifespan because there is no point in having a decade's worth of eggs if a given species' maximum lifespan is measured in months or a few years. Gosden and Telfer (1987) compared ovarian reserve sizes among 19 species of mammals whose maximum adult lifespans (MALS) covered nearly 100 years, and body weights spanned four orders of magnitude (pipistrelle bat to cattle, refer to Table 1). They noted the numbers of PFs in peri-pubertal females scaled allometrically with both body weight $\left(2700 \times \mathrm{M}^{0.47}\right)$ and maximum adult lifespan $\left(820 \times \mathrm{L}^{1.58}\right)$. The authors concluded that larger mammals generally live longer than smaller species and that an increased ovarian reserve size in larger species could be an important adaptation for longer adult lifespans (Gosden \& Telfer 1987).

The naked mole-rat (NMR, Heterocephalus glaber) is an exception to the general rule that small terrestrial mammals have relatively short lifespans (Healy et al. 2014). In fact, NMRs are the longest-lived rodent with a maximum lifespan of 35 years, which is five-fold greater than predicted allometrically for a 35-40 g rodent (Edrey et al. 2011, Ruby et al. 2018). In addition to being renowned for their remarkable longevity, female NMRs show no decline in fertility and fecundity into the third decade of life, and they essentially breed until they die (Buffenstein 2008). Further, NMRs are one of only two eusocial species of mammals (Jarvis \& Bennett 1993), with just one reproductively active female (the queen) in subterranean colonies that number in the tens to hundreds of animals (Jarvis 1991). Whereas all other females within a colony are reproductively suppressed by the queen's aggressive behaviors, these subordinate females can become reproductively activated when removed from the colony and the queen's suppression. Whilst under the queen's suppression, subordinate females maintain a hypogonadotropic-hypooestrogenicanovulatory state (Margulis et al. 1995, Clarke \& Faulkes 1997, Holmes et al. 2009, Swift-Gallant et al. 2015,

Table 1 List of species with their code numbers, orders and numbers of primordial follicles.

\begin{tabular}{|c|c|c|c|c|c|c|}
\hline $\begin{array}{l}\text { Code } \\
\text { No. }\end{array}$ & Species & Order & $\begin{array}{l}\text { No. primordial } \\
\text { follicles* }\end{array}$ & $\begin{array}{c}\% \text { of follicles } \\
\text { growing }\end{array}$ & Body mass $(\mathrm{kg})^{\S}$ & $\underset{\text { lifespan }(y)^{\S}}{\text { Maximum }}$ \\
\hline 1 & Bandicoot (Isoodon macrourus) $^{\ddagger}$ & Marsupialia & 12,440 & 4.9 & 1.600 & 6.8 \\
\hline 2 & Common shrew (Sorex araneus) & Insectivora & 3560 & - & 0.009 & 3.2 \\
\hline 3 & Pipistrelle bat (Pipistrellus pipistrellus) ${ }^{\ddagger}$ & Chiroptera & 3268 & 7.2 & 0.005 & 16.6 \\
\hline 4 & $\begin{array}{l}\text { Greater horseshoe bat (Rhinolophus } \\
\text { ferrumequinum) }^{\ddagger}\end{array}$ & Chiroptera & 7950 & - & 0.023 & 30.5 \\
\hline 5 & House mouse (Mus musculus) & Rodentia & 4270 & 16.5 & 0.021 & 4.0 \\
\hline 6 & Wood mouse (Apodemus sylvaticus) & Rodentia & 3170 & 3.2 & 0.023 & 6.3 \\
\hline 7 & Bank vole (Clethrionomys glareolus) & Rodentia & 4380 & 5.6 & 0.021 & 4.9 \\
\hline 8 & Field vole (Microtus agrestis) & Rodentia & 2858 & 5.0 & 0.046 & 4.8 \\
\hline 9 & Norway rat (Rattus norvegicus) & Rodentia & 5180 & - & 0.300 & 3.8 \\
\hline 10 & Guinea pig (Cavia porcellus) & Rodentia & 29,200 & - & 0.728 & 12.0 \\
\hline 11 & European rabbit (Oryctolagus cuniculus) & Lagomorpha & 75,120 & 4.0 & 1.800 & 9.0 \\
\hline 12 & Domestic cat (Felis catus) & Carnivora & 74,520 & 2.1 & 3.900 & 30.0 \\
\hline 13 & Domestic dog (Canis familiaris) & Carnivora & 150,380 & 19.8 & 40.000 & 24.0 \\
\hline 14 & Sheep (Ovis aries) & Artiodactyla & 105,450 & 0.7 & 80.000 & 22.8 \\
\hline 15 & Swine (Sus scrofa) & Artiodactyla & 420,000 & - & 130.000 & 27.0 \\
\hline 16 & Cattle (Bos taurus) & Artiodactyla & 210,000 & - & 750.000 & 20.0 \\
\hline 17 & Common marmoset (Callithrix jacchus) $)^{\ddagger}$ & Primates & 17,220 & 10.9 & 0.255 & 22.8 \\
\hline 18 & Rhesus monkey (Macaca mulatta) & Primates & 100,000 & 16.1 & 8.235 & 40.0 \\
\hline 19 & Human (Homo sapiens) & Primates & 302,000 & 4.0 & 62.035 & 122.5 \\
\hline NMR & Naked mole-rat (Heterocephalus glaber) & Rodentia & 108,588 & 3.0 & 0.035 & 31.0 \\
\hline
\end{tabular}

Adapted from Gosden and Telfer (1987), with the naked mole-rat added. Body mass and maximum lifespan values are from the AnAge database. Bold values are indicative of species for which the data listed in the AnAge database did not meet the inclusion criteria set by Healy et al. (2014).

*Mean number per pair of ovaries (Gosden \& Telfer 1987); ${ }^{*}$ Fraction (\%) of total follicles that are in the growing stages; ${ }^{\circledR}$ Body mass and

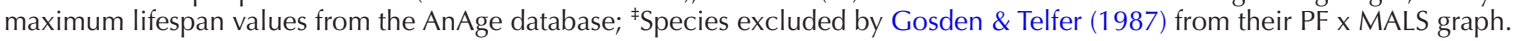


Faykoo-Martinez et al. 2018). Thus, NMRs provide the opportunity to investigate mechanisms critical to reproductive longevity with unprecedented internal biological controls. First, they allow dissociation of the relative importance of longevity and body size for determining the ovarian reserve and, second, they allow dissociation of chronological age and reproductive maturation.

While the histological images of NMR ovaries reported by Kayanja and Jarvis (1971) suggested an unusually large number of primordial follicles, to our knowledge, the size of the ovarian reserve in NMRs has not been previously enumerated or reported. The earliest age at which a subordinate female NMR can be reproductively activated is 6 months (Buffenstein et al. 2012). As such, we counted the ovarian reserve of PFs in NMRs of this age in order to make comparisons with the 19 mammalian species examined by Gosden and Telfer (1987), who evaluated ovaries from peripubertal females. Additionally, in the process of evaluating ovaries from 6-month-old NMRs, we noted the presence of germ cell nests. Lei and Spradling (2013) defined a germ cell nest as 'germ cells that clump morphologically; the interconnected nature of such cells cannot be determined from morphological observation', and their definition applies to our observations in NMRs. Breakdown of germ cell nests is typically complete long before 6 months of age in other mammalian species including mouse and human (Pepling 2006, Hartshorne et al. 2009, Findlay et al. 2015), and nests are absent in adult ovaries. Having observed germ cell nests in ovaries from 6-month-old NMRs, we interrogated ovaries from young adult females (1.25-3.75 years of age) to determine whether germ cell nests are present in both reproductively suppressed and reproductively activated NMRs. Herein, we show that long lifespan and protracted female fertility are associated with an exceptionally large ovarian reserve in the NMR relative to its small body size, and that germ cell nests are present in ovaries of young adults, regardless of reproductive status.

\section{Methods}

\section{Animals}

All experimental procedures followed federal and institutional guidelines and were approved by the University of Toronto Animal Care Committee. Naked mole-rat colonies were housed in polycarbonate cages of three sizes (large: $65 \mathrm{~cm}$ $\mathrm{L} \times 45 \mathrm{~cm} \mathrm{~W} \times 23 \mathrm{~cm} \mathrm{H}$; medium: $46 \mathrm{~cm} \mathrm{~L} \times 24 \mathrm{~cm} \mathrm{~W} \times 15$ $\mathrm{cm} \mathrm{H}$; small: $30 \mathrm{~cm} \mathrm{~L} \times 18 \mathrm{~cm} \mathrm{~W} \times 13 \mathrm{~cm} \mathrm{H}$ ) connected by tubes $(25 \mathrm{~cm} \mathrm{~L} \times 8 \mathrm{~cm} \mathrm{D})$ and lined with corn cob bedding. Reproductive activation was achieved by pair housing an adult female with an adult male from a different colony for 4 weeks in a single, medium polycarbonate cage. Naked molerats were kept on a $12 \mathrm{~h}$ light: $12 \mathrm{~h}$ darkness cycle at $28-30^{\circ} \mathrm{C}$ and fed a diet consisting of sweet potato and wet 19\% protein mash ad libitum (Teklad global diet; Envigo).

\section{Ovary collection and processing}

Immediately following euthanasia by isoflurane, ovaries from five 6-month-old NMRs were removed and immersed in 10\% buffered formalin overnight. Until the time of euthanasia, these females had been housed with their natal colony, including their queens, and therefore, they were reproductively suppressed. Ovaries were transferred to $70 \%$ ethanol and shipped overnight on ice packs to Cornell University. Ovaries were then washed three times in $70 \%$ ethanol, embedded in paraffin and serial sectioned at $6 \mu \mathrm{m}$. Every tenth section from one ovary per animal was mounted on glass slides and stained with hematoxylin and eosin (H\&E). Imaging and counting methods are described subsequently.

For young adult NMRs, ovary halves from four sets of agematched subordinate and reproductively activated females were analyzed ( $n=4$ each). These females ranged in age from 1.25 to 3.75 years. The maximum age difference between agematched subordinate and reproductively activated females was 3 months. These young adult females were part of a larger, neurobiology study (Faykoo-Martinez et al. 2018), and their ovaries were dissected and processed in a different manner than the 6-month-old NMRs. Ovaries were dissected, cut in half with a scalpel and immersed in $4 \%$ paraformaldehyde for $1-2 \mathrm{~h}$ and then transferred to PBS- $0.1 \%$ Triton X-100 and stored at $4{ }^{\circ} \mathrm{C}$ until shipped. One half of an ovary was dedicated to this study, and upon delivery, ovary halves were washed three times in $70 \%$ ethanol, embedded in paraffin and serial sectioned at $6 \mu \mathrm{m}$. Every tenth section was mounted on glass slides and $\mathrm{H} \& \mathrm{E}$ stained. Every tenth section from each of eight animals was viewed with a Leica DM 2500 optical microscope and digital images of germ cell nests were obtained with a Leica DFC295 camera (Leica Biosystems). The examination of young adult ovaries for germ cell nests was qualitative rather than quantitative because only approximately one half of each ovary was available for study.

\section{Germ cell counting}

For ovaries from 6-month-old NMRs, digital images of every tenth H\&E stained section were prepared with an Aperio CSO ScanScope (Leica Biosystems) and downloaded to a tablet (Surface Pro, Microsoft, Redmond, WA). The image processing package Fiji for ImageJ (Schindelin et al. 2012) was used to count PFs, germ cells within nests, and growing follicles by ticking off these objects with a stylus. To avoid double counting across sections, only germ cells for which the nucleus was visible were counted. Following the methods of Gosden and Telfer (1987), the total numbers of PFs per animal were estimated by multiplying the average raw counts from one ovary by two, and then by both the sampling frequency (10) and a correction factor as described by Abercrombie (1946). The equation for the correction factor is: (section thickness (6 $\mu \mathrm{m}) \div$ (section thickness $(6 \mu \mathrm{m})+$ average germ cell nucleus diameter $(18.1 \mu \mathrm{m}))=0.25)$. We measured oocyte nuclear diameters in 10 respresentative PFs from each animal using Fiji to calculate the average diameter of $18.1 \mu \mathrm{m}$.

To calculate the predicted size of the NMR ovarian reserve based on their body weight, we first used the power function reported by Gosden and Telfer (1987) for 19 species of 
mammals. We also calculated the predicted size of the NMR ovarian reserve based on our value for MALS in the NMR and using the Gosden and Telfer power function, which excluded the bandicoot, pipistrelle and greater horseshoe bats, and common marmoset. Because Gosden and Telfer (1987) did not include the raw data that they used to generate their power functions involving body weight and MALS, we searched the AnAge database for the body masses and maximum lifespans (MaxLS) of the same 19 mammalian species, plus the NMR (https:// genomics.senescence.info/species/; accessed 4 May 2020). We used the AnAge values to produce linear log-log regression lines and power functions $\left(Y=a X^{b}\right)$ for the numbers of PFs in the 19 species (JMP, SAS, Cary, NC). We similarly generated power functions after excluding the four species that Gosden and Telfer (1987) excluded from their analysis of PF x MALS to determine how the predicted numbers of PFs in NMRs compared with the results derived from the AnAge values. We also generated power functions after limiting our analysis to the 10 Gosden and Telfer species that had high-quality AnAge data for body mass and MaxLS, using the parameters for data quality set forth by Healy et al. (2014). Their exclusion criteria for MaxLS included those species with less than 10 longevity records, or with low or questionable data quality as defined by the AnAge database (de Magalhães et al. 2007, de Magalhães \& Costa 2009). Healy et al. (2014) included the NMR in their report of lifespan variation in birds and mammals, and we entered the AnAge values for NMR body mass and MaxLS into all of the power functions that we generated using the AnAge database.

\section{Immunofluorescent imaging of germ cell nests}

To enhance the visualization of germ cell nests in ovaries from 6-month-old and young adult females, some of the remaining sections underwent immunofluorescence for the germ cell marker DDX4 (a.k.a. mouse vasa homolog (MVH)) and YBOX2 (Y box protein 2, a.k.a. (MSY2), which is present exclusively in diplotene-stage and mature oocytes (Gu et al 1998). Slides were deparaffinized and rehydrated with three washes of Safeclear (Fischer Scientific) for $10 \mathrm{~min}$ each, followed by three washes of each concentration in a graded series of ethanol $(100,95,80,70 \%)$ for 5 min. After twice rinsing the slides for $5 \mathrm{~min}$ in distilled water, the slides were incubated in $10 \mathrm{mM}$ sodium citrate $(\mathrm{pH} 6.0)$ for $40 \mathrm{~min}$ at $95^{\circ} \mathrm{C}$. Permeabilization was performed in $0.2 \%$ Triton-X 100 in PBS for $1 \mathrm{~h}$. Sections were blocked for $4 \mathrm{~h}$ in blocking solution $(2.52 \mathrm{mg} / \mathrm{mL}$ glycine, 10\% goat serum, 3\% BSA, 0.2\% Tween20) and then incubated overnight at room temperature (RT) in 1:100 dilution of rabbit anti-DDX4 or anti-YBOX2 polyclonal antibody (Abcam 13840; Sigma SAB4502102, respectively) in blocking solution. After two 10-minute washes with $0.1 \%$ Tween20 in PBS (PBS-Tw20), the slides were incubated in goat anti-rabbit secondary antibody AlexaFluor $(594$ and goat antirabbit AlexaFluor 488 (Jackson ImmunoResearch) for $2 \mathrm{~h}$ at RT. The slides were twice rinsed with PBS-Tw20 for 5 min, counterstained with DAPI and mounted in Vectashield (Vector Laboratories, Burlingame, CA). Sections containing germ cell nests were imaged with a Zeiss Axiolmager M2 microscope and Axiocam 506, and the images were processed using Zen 2 (Carl Zeiss AG).

\section{Results}

Six-month-old NMRs had an average ( \pm S.D.) of 108,588 $( \pm 69,890)$ PFs (Table 1$)$, and oocytes within nests accounted for $1.4 \%( \pm 0.4 \%)$ of the non-growing germ cells. Growing follicles accounted for $3 \%( \pm 1.1 \%)$ of the total number of follicles, which is the lowest fraction of growing follicles amongst the four Rodentia species for which Gosden and Telfer (1987) enumerated the fractions of growing follicles (Table 1). Based on an average body mass of $35 \mathrm{~g}$, the NMR has an exceptionally large ovarian reserve as compared to other similarly sized mammals (Fig. 1A). In comparison to the seven other species weighing less than $100 \mathrm{~g}$ that were evaluated by Gosden and Telfer (1987), the size of the ovarian reserve in NMRs is more than an order of magnitude greater than the species that was closest in body weight to the NMR, that is, the greater horseshoe bat (Rhinolophus ferrumequinum, Fig. 1A). Whereas the guinea pig (Cavia porcellus, \#10 in Fig. 1A) has the largest ovarian reserve $(29,200)$ among the five other rodent species, its body weight is an order of magnitude greater than the NMR.

We calculated the predicted ovarian reserve size for 6-month-old NMRs to be 5,703 PFs based on body mass, using the equation reported by Gosden and Telfer (1987), which is $\mathrm{PF}=27,700 \times \mathrm{M}^{0.47}$. We used body mass data from the AnAge database to produce power functions for PFs and body mass, and either included all 19 species (Fig. 2A) or excluded nine species based on the exclusion criteria used by Healy et al. (2014) (Table 2). The different power functions yielded a relatively narrow range of results (5617-6420 PFs) for the predicted size of the NMR ovarian reserve, which is 16.9- to 21.4-fold less than our result $(108,588)$.

Owing to its eusociality, the age of onset of adulthood in NMRs can be somewhat arbitrary, since all females within a colony, save for the queen, will be reproductively inactive. Whereas 6 months is the youngest age at which female NMRs can be reproductively activated (Buffenstein et al. 2012), not all animals have reached adult body size and the onsets of vaginal patency and ovulatory activity upon removal from the queen's suppression are highly variable between 6 and 12 months of age (Holmes, unpublished observations). Conversely, growth has largely plateaued and onset of reproductive activation is less variable in females at 1 year of age (Swift-Gallant et al. 2015, Faykoo-Martinez et al. 2018), and therefore, for the purpose of determning MALS we have assigned this age as the onset of adulthood regardless of reproductive state,that is, suppressed or activated. Following Gosden and Telfer (1987), we calculated MALS for the NMR to be 34 years, based on the difference between their maximum longevity (35 years) and the youngest age at which we considered them to be adults (1 year). Upon plotting the values for the NMR on the Gosden and Telfer graph for PF numbers vs MALS (Fig. 1B), we find 

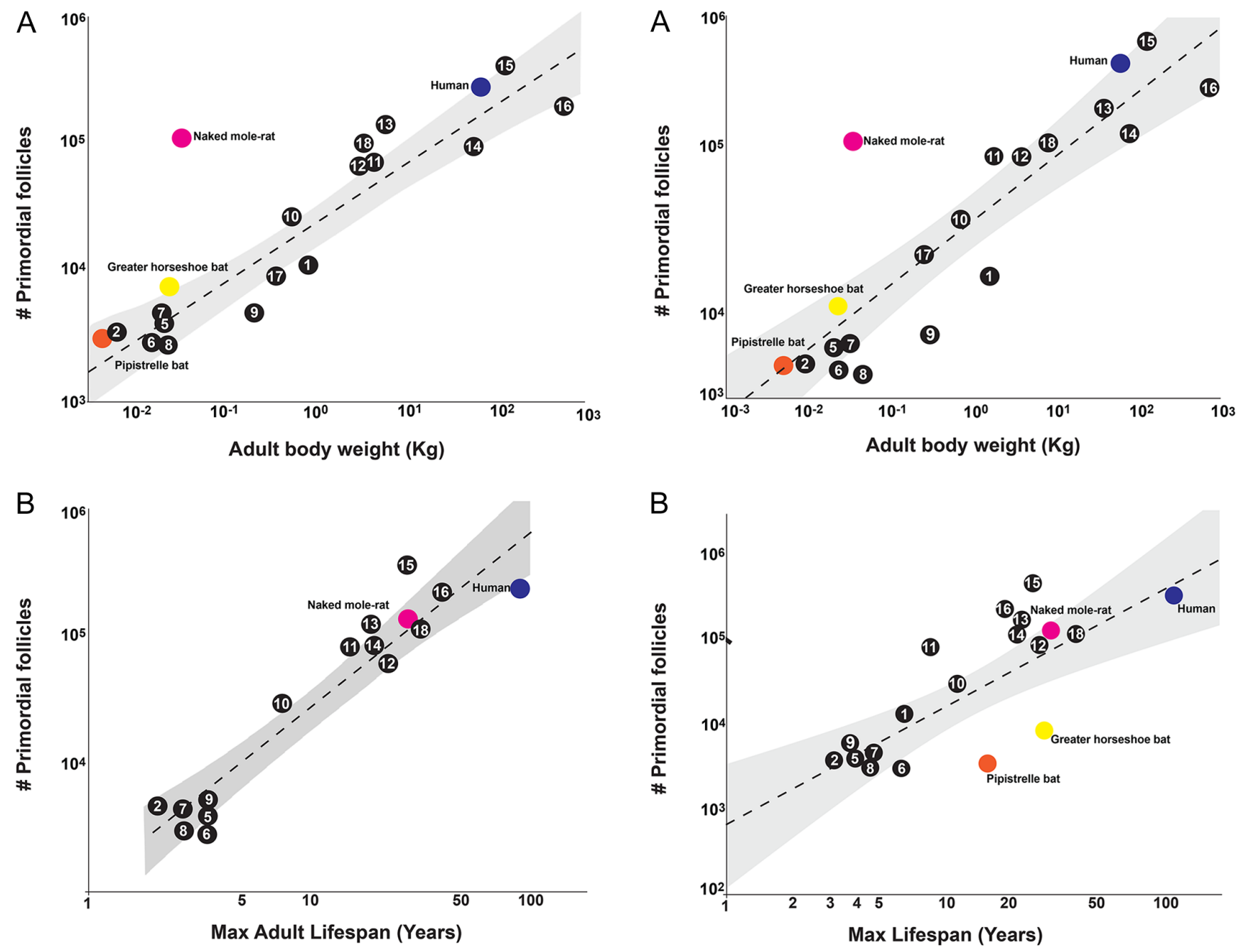

Figure 1 (A) Variation with body weight $(\mathrm{kg})$ in the numbers of primordial follicles per species. (B) Variation with the length of the maximum adult lifespan in the numbers of primordial follicles per species. The linear regressions with $95 \% \mathrm{Cls}$ and the allometric formulas do not include the naked mole-rat data points. Refer to Table 1 for the list of species codes. Adapted with permission from Gosden and Telfer (1987).

the data point for the NMR lies within the $95 \% \mathrm{Cl}$ that was based on results from 15 other mammalian species. We calculated the predicted ovarian reserve size for 6-month-old NMRs to be 215,551 PFs based on MALS using the power function reported by Gosden and Telfer (1987), which is $P F=820 \times L^{1.58}$. Our estimate of MALS for the NMR (34 years) lies closest to the rhesus monkey (Macaca mulatta, \#18 in Fig. 1B), which had an ovarian reserve size of 100,000 PFs and a MALS of 35 years.

We used maximum lifespan (MaxLS) values from the AnAge database, and either included all 19 species (Fig. 2B) or excluded certain species based on the exclusion criteria used by Gosden and Telfer (1987) and by Healy et al. (2014), to produce power functions for PFs and MaxLS (Table 3). The different power functions yielded a

Figure 2 (A) Variation with body weight ( $g$ ) in the numbers of primordial follicles per species. (B) Variation with the length of the maximum lifespan (MaxLS) in the numbers of primordial follicles per species. Values for body weight and MaxLS are from the AnAge database. The linear regressions with $95 \% \mathrm{Cls}$ and the allometric formulas do not include the naked mole-rat data points. Refer to Table 1 for the list of species codes.

broad range of results for the predicted size of the NMR ovarian reserve (51,991-215,551 PFs). When the lifespan data for the pipistrelle and greater horseshoe bats were excluded, the predicted NMR ovarian reserve size was greater than we measured. Conversely, when bat lifespan data were included, the predicted NMR ovarian reserve size was less than we measured (Table 3 ).

Primordial follicles accounted for the vast majority of the ovarian reserves of the 6-month-old NMRs, but germ cell nests can be seen scattered throughout the ovaries (Fig. 3). Generally, each nest contained approximately four germ cells, with nine being the maximum number of germ cells in a single nest. For the young adult NMRs, three of four reproductively suppressed and two of four reproductively activated females had germ cell nests 
Table 2 Predicted size of the ovarian reserve in the naked mole-rat (NMR) using the power function from Gosden \& Telfer (1987) for body mass (BM). The power functions for which values from the AnAge Database were used include the same 19 species reported by Gosden \&Telfer (1987), as well as power functions when the same four species were excluded. Additional power functions were based on the 10 species in common between the Gosden \& Telfer (1987) and Healy et al. (2014) studies. The NMR value for body mass (BM; 35 g) is from the AnAge Database.

\begin{tabular}{|c|c|c|c|c|c|}
\hline Source & $\mathbf{B M}(\mathrm{kg})$ & Formula & Predicted & Actual & Fold difference \\
\hline Gosden \& Telfer (1987) & 0.035 & $27,700 \times M^{0.47}$ & 5703 & 108,588 & $\uparrow 19.0$ \\
\hline AnAge Database & 0.035 & & & & \\
\hline 19 species & & $25,347 \times M^{0.45}$ & 5617 & 108,588 & $\uparrow 19.3$ \\
\hline 15 species $^{\ddagger}$ & & $24,789 \times M^{0.47}$ & 5082 & 108,588 & $\uparrow 21.4$ \\
\hline 10 species $^{\S}$ & & $29,424 \times M^{0.45}$ & 6420 & 108,588 & $\uparrow 16.9$ \\
\hline
\end{tabular}

*Excluded the same four species as Gosden and Telfer (1987), that is, bandicoot, pipistrelle and greater horseshoe bats, and common marmoset. ${ }^{\S}$ Limited to the 10 species' values from the AnAge database that met the inclusion criteria set by Healy et al. (2014).

within their ovaries (Fig. 4). Germ cell nests were present across the age range examined, that is, in females that were 1.25 and 3.75 years of age at the time of collection. In both 6-month-old and young adult females, the germ cells within nests labeled positive for the germ cell marker DDX4 and the oocyte marker MSY2 by immunofluorescence (Figs 3 and 4). Follicle growth had advanced further in the activated than in the suppressed females, and only reproductively activated females showed signs that they had ovulated, that is, presence of corpora lutea and/or corpora albicans (not shown).

\section{Discussion}

In this study, we have determined that the NMR has an exceptionally large ovarian reserve relative to other mammalian species of similar body weight. This is likely to be one of the means by which female NMRs are able to remain fertile and fecund into their third decade of life. However, it remains to be definitively determined if the large ovarian reserve in NMRs at 6 months of age is due to: (1) enhanced mitotic activity of PGCs and oogonia during pre- and/or postnatal life, and/or (2) reduced germ cell atresia during the mitosis-meiosis transition and during nest breakdown/ PF formation. Whereas neo-oogenesis in postnatal and adult mammals is a contentious issue in the field of reproductive biology (Johnson et al. 2004, Begum et al. 2008, Tilly et al. 2009, Albertini \& Gleicher 2015,
Horan \& Williams 2017, Wang et al. 2017), the NMR's large ovarian reserve and protracted fertility warrant consideration for neo-oogenesis in this species. Indeed, the unusual finding of germ cell nests in NMRs that are years old would be consistent with neo-oogenesis, but not proof of it. From the morphological appearance of the germ cell nests in NMR ovaries, we are unable to determine at this time whether the structures formed relatively recently or at a much earlier age, including prenatally, and failed to breakdown and form PFs. Also, it remains to be determined whether the nests in adult NMR ovaries would subsequently breakdown and form PFs that have the capacity to activate, grow, complete meiosis, ovulate, fertilize and produce viable offspring. Advances in folliculogenesis have made it possible to activate PFs and complete the growth and maturation process in vitro (Eppig \& O'Brien 1996, Cortvrindt \& Smitz 2002), and therefore, it might be possible to isolate germ cell nests from adult NMR ovaries and manipulate them in culture to determine whether they form PFs and complete all of the steps necessary for fertilization and the production of viable offspring.

Lei \& Spradling (2013) defined a germline cyst as 'a cluster of interconnected germ cells generated by mitotic divisions with incomplete cytokinesis', and whether adult NMR ovaries contain germline cysts remains to be determined. In the current study, we have classified clusters of germ cells within NMR ovaries as nests based on their morphology and immunoreactivity

Table 3 Predicted size of the ovarian reserve in the naked mole-rat (NMR) using the power function from Gosden and Telfer (1987) for maximum adult lifespan (MALS; 34 years). Naked mole-rat value for MALS is based on our own determination, as described in the text. Gosden \& Telfer (1987) excluded four of 19 species for their MALS formula. The power functions for which values from the AnAge Database were used include the same 19 species reported by Gosden \& Telfer (1987), as well as power functions when the same four species were excluded. Additional power functions were based on the 10 species in common between the Gosden \& Telfer (1987) and Healy et al. (2014) studies. The NMR value for maximum lifespan (MaxLS; 31 years) is from the AnAge Database.

\begin{tabular}{|c|c|c|c|c|c|}
\hline Source & Lifespan, years & Formula & Predicted & Actual & Fold difference \\
\hline Gosden \& Telfer (1987) & $34^{*}$ & $820 \times L^{1.58}$ & 215,551 & 108,588 & $\downarrow(2.0)$ \\
\hline AnAge Database & $31^{+}$ & & & & \\
\hline 19 species & & $712 \times L^{1.35}$ & 72,956 & 108,588 & $\uparrow 1.5$ \\
\hline 15 species $^{\ddagger}$ & & $592 \times L^{1.56}$ & 124,622 & 108,588 & $\downarrow(1.1)$ \\
\hline 10 species $^{\S}$ & & $416 \times L^{1.41}$ & 51,991 & 108,588 & $\uparrow 2.1$ \\
\hline
\end{tabular}

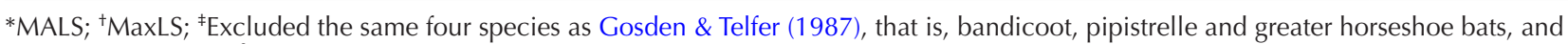
common marmoset; ' $\$$ imited to the 10 species' values from the AnAge database that met the inclusion criteria set by Healy et al. (2014). 

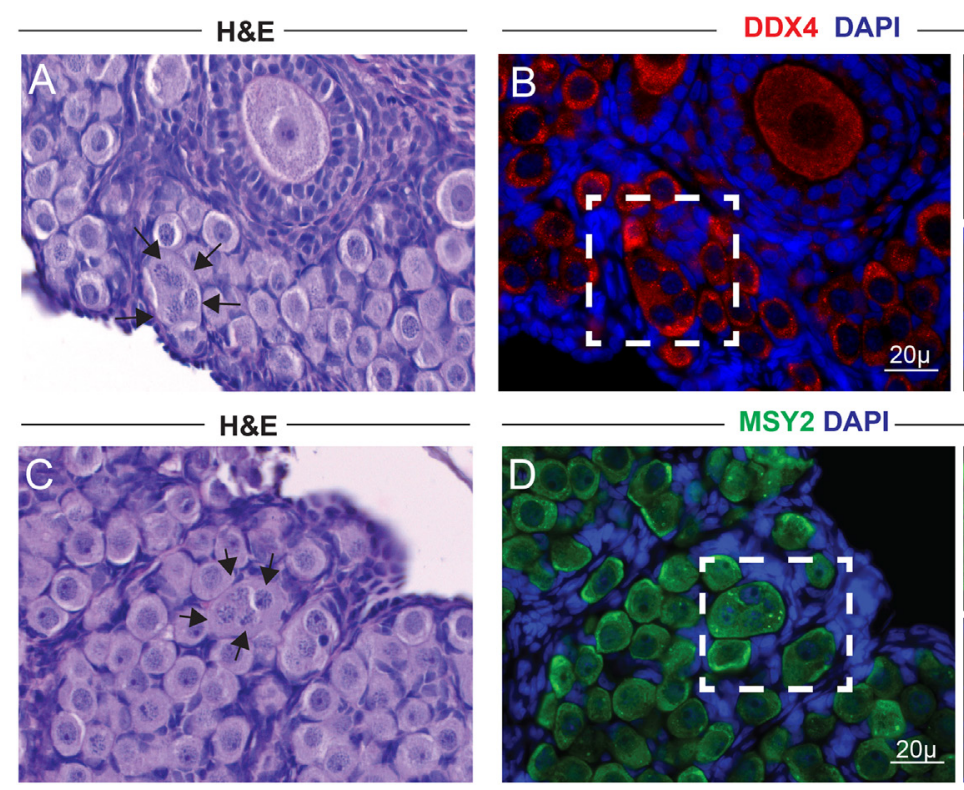

Figure 3 Representative $\mathrm{H} \& \mathrm{E}$ photomicrographs (A and C) of an ovary from a 6-month-old NMR. Arrows point to germ cell nests. (B and D) Representative immunofluorescent images (DDX4 red, MSY2 green, DAPI nuclear stain blue) of germ cell nests. Images in (A) and (B) are from adjacent sections from one region of an ovary, and images in (C) and (D) are from adjacent sections from a different region of an ovary. Arrows within the DAPI only panels point to the corresponding nuclei of the oocytes within the nests. for MSY2, which is present exclusively in diplotenestage and mature oocytes (Gu et al. 1998). As part of a separate study, we attempted to identify interconnected germ cells in embryonic and neonatal NMR ovaries by immunofluorescence for the bridge protein TEX14, but this was unsuccessful because the antibody for TEX14 (Abcam ab41733, Cambridge, MA) did not work in this species. TEX14 is present in intercellular bridges within mouse germline cysts before E14.5 (Greenbaum et al. 2009). If we are able to identify intercellular bridges in embryonic NMR ovaries using different anti-TEX14 antibodies or by electron microscopy, we will then expand our search to include adult ovaries.

Whereas the size of the NMR ovarian reserve appears to be exceptionally large relative to its body weight (Fig. $1 \mathrm{~A})$, the size of the ovarian reserve is unremarkable relative to its adult longevity (Fig. 1B). However, the size of the NMR ovarian reserve might actually be relatively small in relation to its MALS, if the NMR's protracted fertility is taken into consideration. Peri-pubertal NMRs and rhesus monkeys have similar ovarian reserve sizes and MALS, but unlike female rhesus monkeys, female NMRs show no aging-associated decline in fertility and fecundity (Buffenstein 2008). Conversely, captive rhesus monkeys produce offspring well into their twenties, but thereafter they cease to be reproductively active, which results in a post-reproductive lifespan that could last another decade (Gagliardi et al. 2007).

Gosden \& Telfer (1987) did not indicate if the values used for MALS were for mammals in their natural environments or in captivity, however, many of the species in their study are domesticated (e.g. cat, dog, sheep, swine, cattle), and therefore, we assumed the MALS values were based on animals that had been maintained in captivity. We made the same assumption for the NMR and set its MALS to 34 years. Gosden and
Telfer (1987) excluded the bandicoot, marmoset, and the pipistrelle and greater horseshoe bats from their PF vs MALS figure, and they stated, 'In most species, there was sufficient reliable data for estimating the maximum adult lifespan by subtracting the characteristic pubertal age from total longevity'. Presumably, reliable MALS data were not available for these four species at the time of publication. Interestingly, we now know that the pipistrelle and greater horseshoe bats are relatively longlived as compared to rodents and insectivores of similar size (Healy et al. 2014), and it is apparent that the bats' $\mathrm{PF}$ values lie far to the right of the regression line in Fig. 2B. Gosden \& Telfer (1987) speculated that 'these species may depend on parsimonious utilization of follicles in order to prevent premature loss of fecundity'. Whether the NMR has an ovarian reserve that is relatively large or small for its long lifespan appears to depend on which species are included in the allometric analysis, in particular bats (Table 3), and whether lifespans are based on captive or wild data. If it proves to be correct that the NMR has a small ovarian reserve relative to its exceptionally long reproductive lifespan, then this species should also be considered for parsimonious utilization of their germ cells, especially if they do not demonstrate adult neo-oogenesis. Whereas the growing fraction of total follicles was relatively small at $3 \%$ in NMRs (Table 1), this does not necessarily support parsimonious utilization of follicles in this species. Gosden \& Telfer (1987) noted from their data that the 'fraction of growing follicles are far too variable to be useful indicators of the rate of depletion of the follicle store'. The remarkably large number of non-growing PFs in NMR ovaries is likely a major contributor to the low fraction of growing follicles in this species.

There are several different methods for estimating the size of the ovarian reserves in mammals (Tilly 2003), 

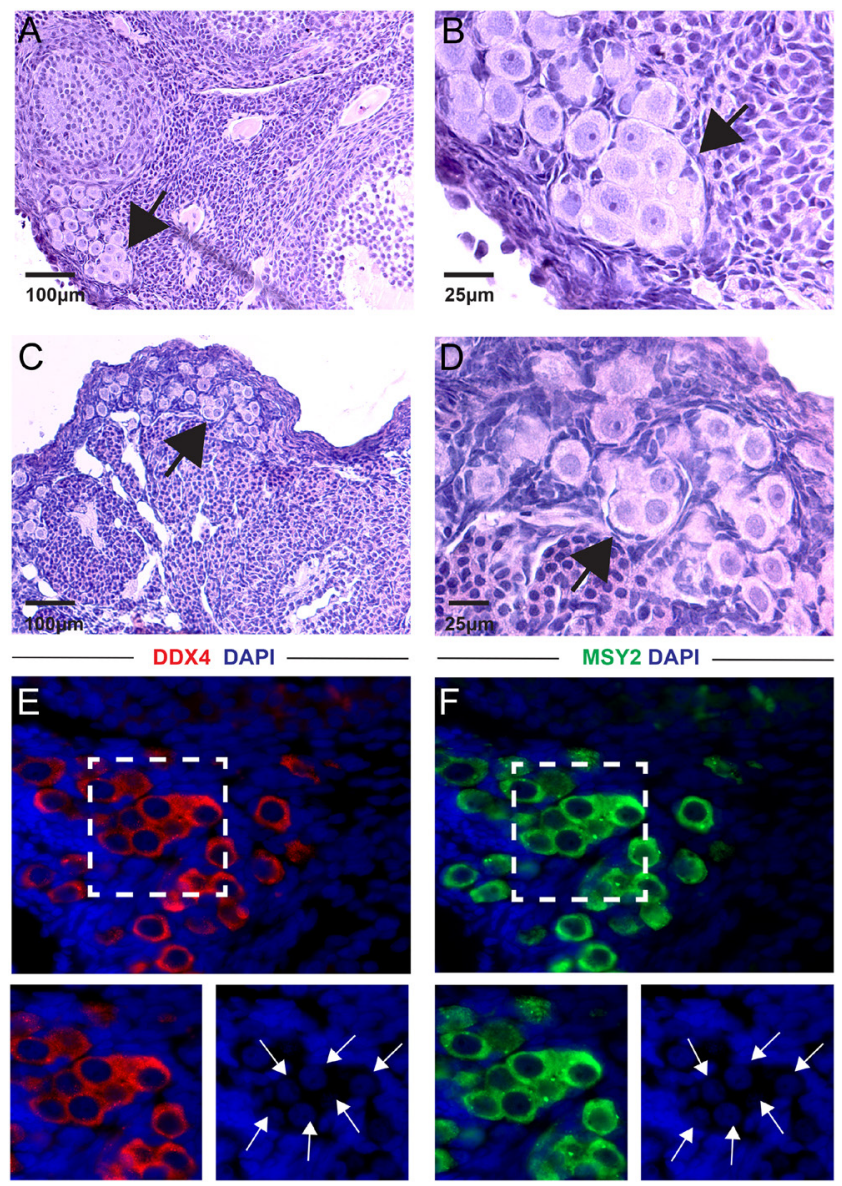

Figure 4 Representative H\&E photomicrographs of ovaries from (A and B) a 2-year, 10-month-old reproductively suppressed female, and (C and D) a 2-year, 7-month-old reproductively activated female. Arrows point to germ cell nests. (E and F) Representative immunofluorescent images (DDX4 red, MSY2 green, DAPI nuclear stain blue) of a germ cell nest in an ovary from a 3-year, 8-month-old reproductively suppressed female. Arrows within the DAPI only panels point to the corresponding nuclei of the oocytes within the nest.

thus, we attempted to follow the method of Gosden \& Telfer (1987) as closely as possible in order to make accurate comparisons among NMRs and the 19 species of mammals that they included in their study. This is not to say that this method is necessarily the optimum way to estimate ovarian reserve size, especially in light of recently developed methods that evaluate whole ovaries rather than ovarian sections of varying thicknesses and sampling frequencies (Faire et al. 2015, Malki et al. 2015, Rinaldi et al. 2018, McKey et al. 2020). These so-called 'whole-mount' immunofluorescent approaches could prove to be more accurate than serial sectioning, though it might be an impractical approach for some species due to ovarian size, consistency, and availability of antibodies with adequate tissue penetration and crossreactivity. Thus far, we have had limited success with NMR ovaries in this regard. Nevertheless, no matter what formula is used to calculate the ovarian reserve size based on counts from serially sectioned NMR ovaries, the raw counts for 6-month-old females from this study $(21,805 \pm 14,034$ PFs per ovary) leave no doubt that this species has an abundance of non-growing germ cells for its body size. In addition to using the Gosden \& Telfer (1987) formula for estimating ovarian reserve size from serial-section counts, we also calculated the size of the NMR ovarian reserve using another method described by Bristol-Gould et al. (2006a). Their method yielded a result for the NMR $(112,332 \pm 71,904)$ that differed from the Gosden and Telfer method by only $3.7 \%$. BristolGould et al. (2006b) estimated the ovarian reserve size in 6-month-old CD1 mice to be $1487 \pm 109$ PFs per animal, a value that is 75 times less than in NMRs of the same chronological age. And whereas 6-monthold mice will soon begin to show signs of reproductive aging within a matter of months (Chiang et al. 2010, Hirshfeld-Cytron et al. 2011, Briley et al. 2016), female NMRs will have their entire decades-long reproductive lifespan ahead of them when they reach this age. The one critically important caveat being that almost all female NMRs will never realize their vast reproductive potential because their queen will prevent them from doing so.

In conclusion, we can now add an exceptionally large ovarian reserve and the presence of germ cell nests in adult ovaries to the NMR's extensive list of remarkable adaptations, which include an unusually long lifespan, tolerance to prolonged anoxia, and near-complete cancer resistance (Tian et al. 2013, Park et al. 2017, Ruby et al. 2018). Certainly, the large ovarian reserve relative to its small body size is one mechanism that can contribute to protracted fertility in long-lived NMRs, but it remains to be determined whether parsimonious germ cell usage and/or adult neo-oogenesis are also contributing factors. Female reproductive lifespans are comparable in NMRs and humans, which are measured in decades in both species. Therefore, elucidating the means by which NMR females escape aging-associated declines in fertility and fecundity might lead to the development of interventions that could preserve fertility as women age.

\section{Declaration of interest}

The authors declare that there is no conflict of interest that could be perceived as prejudicing the impartiality of the research reported.

\section{Funding}

This work was funded by NSERC Discovery Grant RGPIN 201804780, NSERC Discovery Accelerator Supplement RGPAS 2018-522465, and an Ontario Early Researcher Award to M $\mathrm{M} \mathrm{H}$, and NSERC PGS D Scholarship to M F-M. M A B-E was supported by the Eunice Kennedy Shriver National Institute of 
Child Health \& Human Development of the National Institutes of Health under Award Number R00HD090289 to M A B-E and P50HD096723 Pilot Project.

\section{Author contribution statement}

N J P conceived the study, analyzed data, wrote the initial and final drafts of the manuscript. A M P performed H\&E histology, enumerated germ cells, reviewed and edited the manuscript. M F-M collected NMR ovaries, processed and shipped them for histolology, reviewed and edited the manuscript. M A B-E performed immunofluorescence histology, microscopy and imaging, prepared figures, reviewed and edited the manuscript. D F A conceived the study, reviewed and edited the manuscript. M M H conceived the study, provided NMR ovaries, reviewed and edited the manuscript.

\section{Acknowledgements}

The authors thank the Cornell University Histology/Cytology Core Facility for the preparation of paraffin blocks of naked mole-rat ovaries, and Roger Gosden and Evelyn Telfer for their original work on the numbers of primordial follicles in 19 species of mammals.

\section{References}

Abercrombie M 1946 Estimation of nuclear population from microtome sections. Anatomical Record 94 239-247. (https://doi.org/10.1002/ ar.1090940210)

Albertini DF \& Gleicher N 2015 A detour in the quest for oogonial stem cells: methods matter. Nature Medicine 21 1126-1127. (https://doi. org/10.1038/nm.3969)

Begum S, Papaioannou VE \& Gosden RG 2008 The oocyte population is not renewed in transplanted or irradiated adult ovaries. Human Reproduction 23 2326-2330. (https://doi.org/10.1093/humrep/den249)

Briley SM, Jasti S, McCracken JM, Hornick JE, Fegley B, Pritchard MT \& Duncan FE 2016 Reproductive age-associated fibrosis in the stroma of the mammalian ovary. Reproduction 152 245-260. (https://doi. org/10.1530/REP-16-0129)

Bristol-Gould SK, Kreeger PK, Selkirk CG, Kilen SM, Cook RW, Kipp JL, Shea LD, Mayo KE \& Woodruff TK 2006a Postnatal regulation of germ cells by activin: the establishment of the initial follicle pool. Developmental Biology 298 132-148. (https://doi.org/10.1016/j. ydbio.2006.06.025)

Bristol-Gould SK, Kreeger PK, Selkirk CG, Kilen SM, Mayo KE, Shea LD \& Woodruff TK 2006 $b$ Fate of the initial follicle pool: empirical and mathematical evidence supporting its sufficiency for adult fertility. Developmental Biology 298 149-154. (https://doi.org/10.1016/j. ydbio.2006.06.023)

Buffenstein R 2008 Negligible senescence in the longest living rodent, the naked mole-rat: insights from a successfully aging species. Journal of Comparative Physiology B 178 439-445. (https://doi.org/10.1007/ s00360-007-0237-5)

Buffenstein R, Park T, Hanes M \& Artwohl JE 2012 Naked mole rat. In The Laboratory Rabbit, Guinea Pig, Hamster, and Other Rodents, pp. 1055-1074. Eds MA Suckow, KA Stevens \& RP Wilson. Boston: Academic Press.

Chiang T, Duncan FE, Schindler K, Schultz RM \& Lampson MA 2010 Evidence that weakened centromere cohesion is a leading cause of agerelated aneuploidy in oocytes. Current Biology 20 1522-1528. (https:// doi.org/10.1016/j.cub.2010.06.069)

Clarke FM \& Faulkes CG 1997 Dominance and queen succession in captive colonies of the eusocial naked mole-rat, Heterocephalus glaber.
Proceedings of the Royal Society of London: Series B 264 993-1000. (https://doi.org/10.1098/rspb.1997.0137)

Cortvrindt RG \& Smitz JE 2002 Follicle culture in reproductive toxicology: a tool for in-vitro testing of ovarian function? Human Reproduction Update 8 243-254. (https://doi.org/10.1093/humupd/8.3.243)

Coucouvanis EC, Sherwood SW, Carswell-Crumpton C, Spack EG \& Jones PP 1993 Evidence that the mechanism of prenatal germ cell death in the mouse is apoptosis. Experimental Cell Research 209 238-247. (https://doi.org/10.1006/excr.1993.1307)

de Magalhães JP \& Costa J 2009 A database of vertebrate longevity records and their relation to other life-history traits. Journal of Evolutionary Biology 22 1770-1774. (https://doi.org/10.1111/j.1420-9101.2009.01783.x)

de Magalhães JP, Costa J \& Church GM 2007 An analysis of the relationship between metabolism, developmental schedules, and longevity using phylogenetic independent contrasts. Journal of Gerontology: Series A 62 149-160. (https://doi.org/10.1093/gerona/62.2.149)

Edrey YH, Hanes M, Pinto M, Mele J \& Buffenstein R 2011 Successful aging and sustained good health in the naked mole rat: a long-lived mammalian model for biogerontology and biomedical research. ILAR Journal 52 41-53. (https://doi.org/10.1093/ilar.52.1.41)

Eppig JJ \& O'Brien MJ 1996 Development in vitro of mouse oocytes from primordial follicles. Biology of Reproduction 54 197-207. (https://doi. org/10.1095/biolreprod54.1.197)

Faire M, Skillern A, Arora R, Nguyen DH, Wang J, Chamberlain C, German MS, Fung JC \& Laird DJ 2015 Follicle dynamics and global organization in the intact mouse ovary. Developmental Biology 403 69-79. (https://doi.org/10.1016/j.ydbio.2015.04.006)

Faykoo-Martinez M, Monks DA, Zovkic IB \& Holmes MM 2018 Sexand brain region-specific patterns of gene expression associated with socially-mediated puberty in a eusocial mammal. PLOS ONE 13 e0193417. (https://doi.org/10.1371/journal.pone.0193417)

Finch CE \& Kirkwood TBL 2000 Chance, Development, and Aging. New York: Oxford University Press.

Findlay JK, Hutt KJ, Hickey M \& Anderson RA 2015 How is the number of primordial follicles in the ovarian reserve established? Biology of Reproduction 93 111-117. (https://doi.org/10.1095/ biolreprod.115.133652)

Forabosco A \& Sforza C 2007 Establishment of ovarian reserve: a quantitative morphometric study of the developing human ovary. Fertility and Sterility 88 675-683. (https://doi.org/10.1016/j.fertnstert.2006.11.191)

Funkuda T 1976 Ultrastructure of primordial germ cells in human embryo. Virchows Archives B Cell Pathology 20 85-89.

Gagliardi C, Liukkonen JR, Phillippi-Falkenstein KM, Harrison RM \& Kubisch HM 2007 Age as a determinant of reproductive success among captive female rhesus macaques (Macaca mulatta). Reproduction 133 819-826. (https://doi.org/10.1530/REP-06-0323)

Ge W, Li L, Dyce PW, De Felici M \& Shen W 2019 Establishment and depletion of the ovarian reserve: physiology and impact of environmental chemicals. Cellular and Molecular Life Sciences 76 1729-1746. (https:// doi.org/10.1007/s00018-019-03028-1)

Gomes Fernandes M, Bialecka M, Salvatori DCF \& Chuva de Sousa Lopes SM 2018 Characterization of migratory primordial germ cells in the aorta-gonad-mesonephros of a 4.5-week-old human embryo: a toolbox to evaluate in vitro early gametogenesis. Molecular Human Reproduction 24 233-243. (https://doi.org/10.1093/molehr/gay011)

Gore AC, Hall JE \& Hayes FJ 2015 Aging and reproduction. In Knobil and Neill's Physiology of Reproduction, 4th ed., pp. 1661-1693. Eds TM Plant \& AJ Zeleznik. San Diego: Academic Press.

Gosden RG \& Telfer E 1987 Numbers of follicles and oocytes in mammalian ovaries and their allometric relationships. Journal of Zoology $\mathbf{2 1 1}$ 169-175. (https://doi.org/10.1111/j.1469-7998.1987.tb07460.x)

Gosden RG, Laing SC, Felicio LS, Nelson JF \& Finch CE 1983 Imminent oocyte exhaustion and reduced follicular recruitment mark the transition to acyclicity in aging $\mathrm{C} 57 \mathrm{BL} / 6 \mathrm{~J}$ mice. Biology of Reproduction $\mathbf{2 8}$ 255-260. (https://doi.org/10.1095/biolreprod28.2.255)

Greenbaum MP, Iwamori N, Agno JE \& Matzuk MM 2009 Mouse TEX14 is required for embryonic germ cell intercellular bridges but not female fertility. Biology of Reproduction 80 449-457. (https://doi.org/10.1095/ biolreprod.108.070649)

Grive KJ \& Freiman RN 2015 The developmental origins of the mammalian ovarian reserve. Development 142 2554-2563. (https://doi.org/10.1242/ dev.125211) 
Gu W, Tekur S, Reinbold R, Eppig JJ, Choi Y-C, Zheng JZ, Murray MT \& Hecht NB 1998 Mammalian male and female germ cells express a germ cell-specific Y-box protein, MSY2. Biology of Reproduction 59 1266-1274. (https://doi.org/10.1095/biolreprod59.5.1266)

Hartshorne GM, Lyrakou S, Hamoda H, Oloto E \& Ghafari F 2009 Oogenesis and cell death in human prenatal ovaries: what are the criteria for oocyte selection? Molecular Human Reproduction 15 805-819. (https://doi.org/10.1093/molehr/gap055)

Healy K, Guillerme T, Finlay S, Kane A, Kelly SBA, McClean D, Kelly DJ, Donohue I, Jackson AL \& Cooper N 2014 Ecology and mode-oflife explain lifespan variation in birds and mammals. Proceedings of the Royal Society B 281 20140298. (https://doi.org/10.1098/ rspb.2014.0298)

Hirshfeld-Cytron JE, Duncan FE, Xu M, Jozefik JK, Shea LD \& Woodruff TK 2011 Animal age, weight and estrus cycle stage impact the quality of in vitro grown follicles. Human Reproduction 26 2473-2485. (https://doi. org/10.1093/humrep/der183)

Holmes MM, Goldman BD, Goldman SL, Seney ML \& Forger NG 2009 Neuroendocrinology and sexual differentiation in eusocial mammals. Frontiers in Neuroendocrinology 30 519-533. (https://doi.org/10.1016/j. yfrne.2009.04.010)

Horan CJ \& Williams SA 2017 Oocyte stem cells: fact or fantasy? Reproduction 154 R23-R35. (https://doi.org/10.1530/REP-17-0008)

Jarvis JUM 1991 Reproduction of naked mole-rats. In The Biology of the Naked Mole-Rat, pp. 384-425. Eds JW Sherman, J Jarvis \& RD Alexander. Princeton, NJ: Princeton University Press.

Jarvis JUM \& Bennett NC 1993 Eusociality has evolved independently in two genera of bathyergid mole-rats - but occurs in no other subterranean mammal. Behavioral Ecology and Sociobiology 33 253-260. (https://doi. org/10.1007/BF02027122)

Johnson J, Canning J, Kaneko T, Pru JK \& Tilly JL 2004 Germline stem cells and follicular renewal in the postnatal mammalian ovary. Nature 428 145-150. (https://doi.org/10.1038/nature02316)

Kayanja FIB \& Jarvis J 1971 Histological observations on the ovary oviduct and uterus of the naked-mole-rat. Zeitschrift für Säugetierkunde $\mathbf{3 6}$ 114-121.

Lei L \& Spradling AC 2013 Mouse primordial germ cells produce cysts that partially fragment prior to meiosis. Development 140 2075-2081. (https://doi.org/10.1242/dev.093864)

Malki S, Tharp ME \& Bortvin A 2015 A whole-mount approach for accurate quantitative and spatial assessment of fetal oocyte dynamics in mice. Biology of Reproduction 93 113. (https://doi.org/10.1095/ biolreprod/115.132188)

Mamsen LS, Lutterodt MC, Andersen EW, Byskov AG \& Andersen CY 2011 Germ cell numbers in human embryonic and fetal gonads during the first two trimesters of pregnancy: analysis of six published studies. Human Reproduction 26 2140-2145. (https://doi.org/10.1093/humrep/der149)

Margulis SW, Saltzman W \& Abbott DH 1995 Behavioral and hormonal changes in female naked mole-rats (Heterocephalus glaber) following removal of the breeding female from a colony. Hormones and Behavior 29 227-247. (https://doi.org/10.1006/hbeh.1995.1017)

McKey J, Cameron LA, Lewis D, Batchvarov IS \& Capel B 2020 Combined iDISCO and CUBIC tissue clearing and lightsheet microscopy for in toto analysis of the adult mouse ovary. Biology of Reproduction 102 1080-1089. (https://doi.org/10.1093/biolre/ioaa012)

Monget P, Bobe J, Gougeon A, Fabre S, Monniaux D \& Dalbies-Tran R 2012 The ovarian reserve in mammals: a functional and evolutionary perspective. Molecular and Cellular Endocrinology 356 2-12. (https:// doi.org/10.1016/j.mce.2011.07.046)

Motta PM \& Makabe S 1986a Elimination of germ cells during differentiation of the human ovary: an electron microscopic study. European Journal of Obstetrics and Gynecology and Reproductive Biology 22 271-286. (https://doi.org/10.1016/0028-2243(86)90115-2)

Motta PM \& Makabe S $1986 b$ Germ cells in the ovarian surface during fetal development in humans. A three-dimensional microanatomical study by scanning and transmission electron microscopy. Journal of Submicroscopic Cytology and Pathology 18 271-290.

Motta PM, Makabe S \& Nottola SA 1997 The ultrastructure of human reproduction. I. The natural history of the female germ cell: origin, migration and differentiation inside the developing ovary. Human Reproduction Update 3 281-297. (https://doi.org/10.1093/ humupd/3.3.281)

Myers M, Morgan FH, Liew SH, Zerafa N, Gamage TU, Sarraj M, Cook M, Kapic I, Sutherland A, Scott CL et al. 2014 PUMA regulates germ cell loss and primordial follicle endowment in mice. Reproduction 148 211-219. (https://doi.org/10.1530/REP-13-0666)

Park TJ, Reznick J, Peterson BL, Blass G, Omerbašić D, Bennett NC, Kuich PHJL, Zasada C, Browe BM, Hamann W et al. 2017 Fructosedriven glycolysis supports anoxia resistance in the naked mole-rat. Science 356 307-311. (https://doi.org/10.1126/science.aab3896)

Pepling ME 2006 From primordial germ cell to primordial follicle: mammalian female germ cell development. Genesis 44 622-632. (https://doi.org/10.1002/dvg.20258)

Pereda J, Zorn T \& Soto-Suazo M 2006 Migration of human and mouse primordial germ cells and colonization of the developing ovary: an ultrastructural and cytochemical study. Microscopy Research and Technique 69 386-395. (https://doi.org/10.1002/jemt.20298)

Rinaldi VD, Bloom JC \& Schimenti JC 2018 Whole mount immunofluorescence and follicle quantification of cultured mouse ovaries. Journal of Visualized Experiments 135 e57593. (https://doi. org/10.3791/57593)

Rodrigues P, Limback D, McGinnis LK, Plancha CE \& Albertini DF 2009 Multiple mechanisms of germ cell loss in the perinatal mouse ovary. Reproduction 137 709-720. (https://doi.org/10.1530/REP-08-0203)

Ruby JG, Smith M \& Buffenstein R 2018 Naked mole-rat mortality rates defy Gompertzian laws by not increasing with age. elife 7 e31157. (https://doi.org/10.7554/eLife.31157)

Schindelin J, Arganda-Carreras I, Frise E, Kaynig V, Longair M, Pietzsch T, Preibisch S, Rueden C, Saalfeld S, Schmid B et al. 2012 Fiji: an opensource platform for biological-image analysis. Nature Methods 9 676-682. (https://doi.org/10.1038/nmeth.2019)

Swift-Gallant A, Mo K, Peragine DE, Monks DA \& Holmes MM 2015 Removal of reproductive suppression reveals latent sex differences in brain steroid hormone receptors in naked mole-rats, Heterocephalus glaber. Biology of Sex Differences 6 31. (https://doi.org/10.1186/s13293015-0050-x)

Tian X, Azpurua J, Hine C, Vaidya A, Myakishev-Rempel M, Ablaeva J, Mao Z, Nevo E, Gorbunova V \& Seluanov A 2013 High-molecular-mass hyaluronan mediates the cancer resistance of the naked mole rat. Nature 499 346-349. (https://doi.org/10.1038/nature12234)

Tilly JL 2003 Ovarian follicle counts - not as simple as 1,2, 3. Reproductive Biology and Endocrinology 1 11. (https://doi.org/10.1186/1477-78271-11)

Tilly JL, Niikura Y \& Rueda BR 2009 The current status of evidence for and against postnatal oogenesis in mammals: a case of ovarian optimism versus pessimism? Biology of Reproduction 80 2-12. (https://doi. org/10.1095/biolreprod.108.069088)

Tingen CM, Bristol-Gould SK, Kiesewetter SE, Wellington JT, Shea L \& Woodruff TK 2009 Prepubertal primordial follicle loss in mice is not due to classical apoptotic pathways. Biology of Reproduction 81 16-25. (https://doi.org/10.1095/biolreprod.108.074898)

Wallace WHB \& Kelsey TW 2010 Human ovarian reserve from conception to the menopause. PLOS ONE 5 e8772. (https://doi.org/10.1371/journal. pone.0008772)

Wang N, Satirapod C, Ohguchi Y, Park ES, Woods DC \& Tilly JL 2017 Genetic studies in mice directly link oocytes produced during adulthood to ovarian function and natural fertility. Scientific Reports 710011. (https://doi.org/10.1038/s41598-017-10033-6)

Received 1 June 2020

First decision 30 June 2020

Revised Manuscript received 29 September 2020

Accepted 5 November 2020 